\title{
Особенности баллистического транспорта электронов через открытые двухуровневые системы в сильном высокочастотном электрическом поле
}

\author{
(C) А.Б. Пашковский \\ $\mathrm{AO}, \mathrm{HПП} \mathrm{«ИСТОК»} \mathrm{им.} \mathrm{Шокина“,}$ \\ 141195 Фрязино, Московская область, Россия \\ E-mail: solidstate10@mail.ru
}

(Получена 12 ноября 2015 г. Принята к печати 19 октября 2016 г.)

\begin{abstract}
Для несимметричных дувухбарьерных и трехбарьерных резонансно-туннельных структур с тонкими высокими барьерами, образующих открытые двухуровневые системы, получены аналитические выражения для коэффициентов прохождения и отражения электронов в сильном высокочастотном электрическом поле с частотой, близкой к резонансной. Исследованы зависимости ширины и формы резонансных уровней от амплитуды высокочастотного поля. Показано, что для таких структур практически всегда существуют условия, при которых нерезонансные каналы рассеяния вблизи квантовых уровней могут становиться абсолютно прозрачными.
\end{abstract}

DOI: 10.21883/FTP.2017.05.44419.8111

\section{1. Введение}

Квантовая интерференция лежит в основе многих фундаментальных физических процессов, одним из которых является резонансное туннелирование электронов через многобарьерные структуры. Этот процесс имеет массу интересных особенностей и в статике, и в присутствии высокочастотного электрического поля [1-4], как в строго резонансном случае, так и при частотах, близких к резонансным частотам переходов электронов внутри структуры [5-7]. При этом возможны две принципиально разные ситуации, определяемые в основном толщинами барьеров и температурой системы: резонансное туннелирование может быть когерентным и некогерентным. При толстых барьерах и высоких температурах время жизни электронов на уровне оказывается много больше характерного времени взаимодействия с фононами, когерентность нарушается, и именно процессы рассеяния на фононах оказывают определяющее влияние на поведение электронов и их взаимодействие с СВЧ полем. В принципе, охлаждая систему до сверхнизких температур (в пределе до абсолютного нуля), электронный транспорт всегда можно сделать когерентным, однако для практического использования, да и для экспериментальных исследований интерес представляют температуры не ниже температуры жидкого азота.

С уменьшением толщины барьеров температура, при которой начинается нарушение когерентности, возрастает. Современные технологии давно позволяют создавать барьеры столь малой толщины $[8,9]$, что электронный транспорт через резонансные структуры вообще может быть баллистическим в достаточно широком интервале изменения толщин барьеров даже при комнатных температурах. Особый интерес эта ситуация приобретает для квантовых каскадных лазеров $[10,11]$, которые при когерентном транспорте электронов по оценкам могут иметь квантовую эффективность на уровне десятков процентов [5], в то время как квантовая эффективность одной рабочей ячейки лазера на межподзонных переходах, работающего в режиме некогерентного транспорта электронов, когда на каждый излучательный переход электронов между рабочими уровнями (подзонами) активного участка полупроводниковой структуры приходится несколько безызлучательных переходов, вызываемых фононами, обычно не превосходит долей процента [12-15].

Когерентное туннелирование имеет много интересных особенностей, часть из которых до конца не изучена, особенно в условиях, когда частоты высокочастотных полей и энергии электронов не являются строго резонансными. Известно, что одно- и двухфотонные каналы рассеяния в двух- и трехбарьерных структурах при баллистическом транспорте через центры уровней в сильном поле могут становиться абсолютно прозрачными $[3,16]$. Известно, что вероятность малосигнальных переходов строго следует за формой резонансных уровней, а ширины уровней могут зависеть от амплитуды приложенного поля [5-7]. Особенности резонансных переходов электронов при баллистическом транспорте вблизи центров резонансных уровней в двухбарьерных структурах частично были исследованы в работе [17], а оригинальное физическое объяснение наблюдаемых явлений дано в [18]. Однако рассмотренные ситуации представляют собой лишь частный случай гораздо более широкого класса двухуровневых систем, многие из которых могут представлять гораздо больший интерес для практики, чем обычные двухбарьерные структуры. Кроме теоретического интереса, существует сугубо практический вопрос: как точно должен быть настроен резонатор и насколько может отличаться энергия электронов от строго резонансной, для того чтобы такие структуры при использовании их в качестве активных 


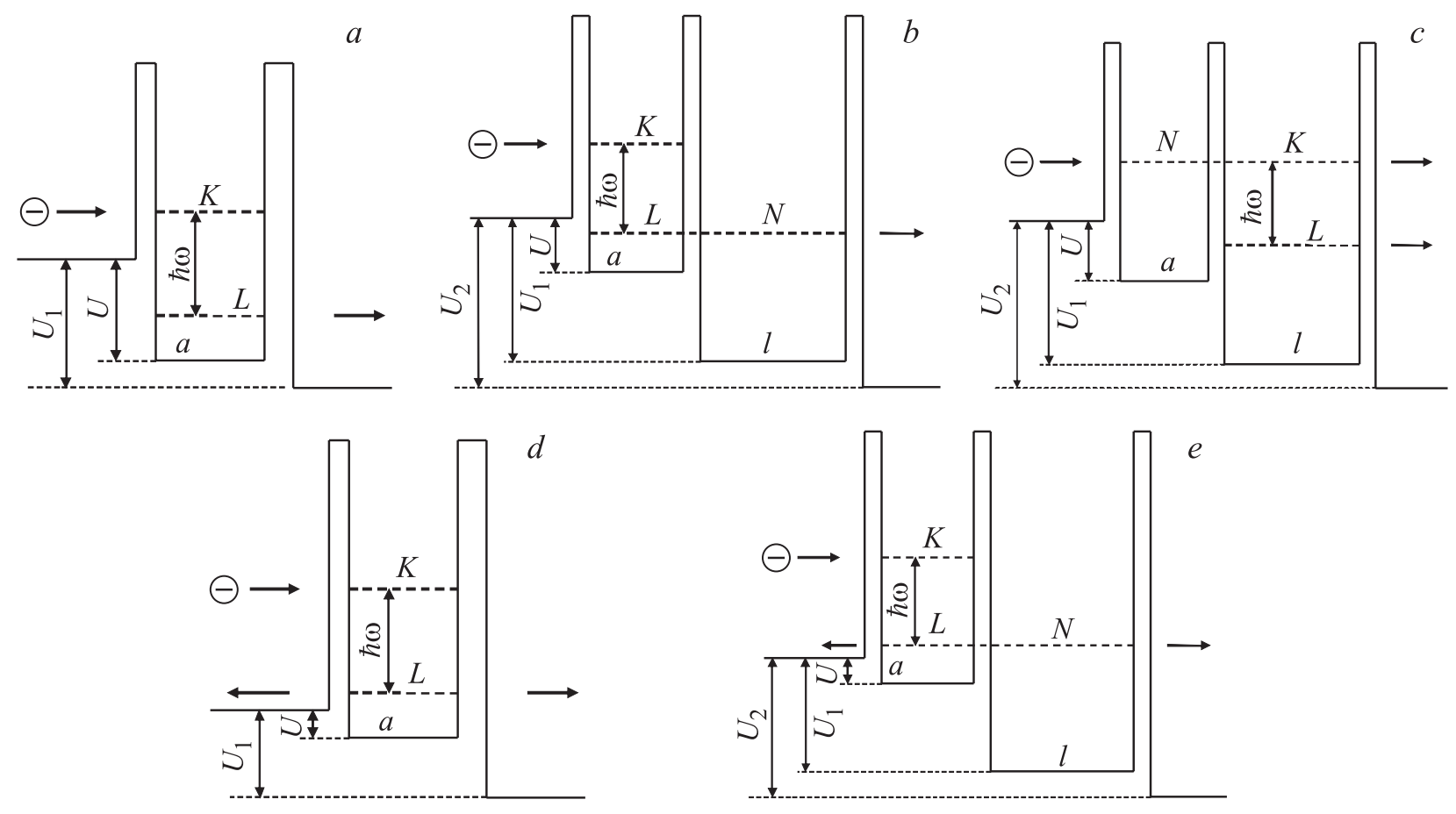

Рис. 1. Схематические зонные диаграммы рассматриваемых двух- и трехбарьерных структур.

ячеек квантовых каскадных лазеров с баллистическим транспортом электронов [11] продолжали достаточно эффективно преобразовывать энергию электронов в СВЧ колебания.

\section{2. Расчет волновых функций}

При униполярных переходах открытая двухуровневая система (электроны могут свободно входить в систему и покидать ее, в этом смысле мы имеем не настоящие уровни, а квазиуровни - максимумы коэффициентов прохождения или взаимодействия с СВЧ полем) может быть реализована различными способами, как в двухбарьерных, так и трехбарьерных структурах, причем в зависимости от того, по какому уровню, нижнему или верхнему, электроны попадают в структуру, переходы будут происходить с поглощением или испусканием фотонов. Для практики в сильных СВЧ полях основной интерес представляют переходы с испусканием фотонов. При этом как для двухбарьерных, так и для трехбарьерных структур возможны две основные ситуации (для определенности считаем, что электроны двигаются слева направо и попадают на верхний уровень структуры): 1) переходы совершаются на уровень, который лежит ниже дна зоны проводимости левого материала и выше дна зоны проводимости правого (рис. 1, $a, b$ ) и могут уходить по нему только направо; ситуация на рис. 1, $c$ аналогична; надо отметить, что положение дна зоны проводимости слева от структуры для случая $1, c$ не актуально; 2) переходы совершаются между уровня- ми, которые лежат выше дна зоны проводимости как левого, так и правого полупроводникового материала (рис. $1, d, e)$, поэтому у электронов есть еще и возможность уйти и налево (отразиться от структуры, испустив фотон).

Рассмотрим структуры с тонкими $\delta$-образными барьерами, к которым приложено однородное высокочастотное электрическое поле $E(t)=2 E \cos \omega t$ $=E\left(e^{i \omega t}+e^{-i \omega t}\right)$. Приближение $\delta$-барьеров [19], интенсивно используемое в данной области [3-7,15-18], очень удобно тем, что резко упрощает решение подобных задач и даже для трехбарьерных структур позволяет получать аналитические решения [16]. Кроме того, для ряда реальных барьеров [20] оно вообще часто является достаточно точным. Рассеянием внутри структуры, как и неравновесными эффектами в контактных областях [21-23], пренебрегается. Пусть моноэнергетический поток электронов с энергией $\varepsilon$ падает слева на резонансный уровень с номером $K$ на расстоянии $\delta \varepsilon$ от его центра, частота $\omega$ на величину $\Delta \omega$ отличается от частоты, строго соответствующей резонансным переходам между центрами $K$ - и $L$-уровней этой структуры (рис. 1$)$.

В двухбарьерных структурах электроны могут сразу уходить из структуры по резонансному уровню. Среди трехбарьерных структур будем рассматривать только такие, в которых один из резонансных уровней второй двухбарьерной структуры с номером $N$ находится вблизи уровня $L$ первой, образуя общий расщепленный (двукратно вырожденный) резонансный уровень всей структуры, по которому электроны и уходят направо. Для простоты будем обозначать его индексом $L N$. В дальней- 
шем предполагается, что расстояние между расщепленными уровнями намного больше ширины уровней $\Gamma_{L N}$ и переходы осуществляются только на один из этих уровней, причем для дальнейших расчетов неважно, куда переходят электроны с самого верхнего уровня, на верхний или на нижний расщепленный уровень, несмотря на ортогональность волновых функций на них. Дело в том, что в первой яме форма волновой функции на каждом из расщепленных уровней совпадает, а во второй яме отличается только фазовым множителем -1 , который никак не сказывается на вероятности переходов. В принципе вполне возможна ситуация, когда необходимо учитывать наличие сразу обоих уровней, однако она принципиально отличается от рассматриваемой и требует специального исследования. По этой же причине не рассматривается и симметричная трехбарьерная структура с расщепленными уровнями [23]. Кроме того, двухуровневая система может быть и в трехбарьерной структуре со сквозным (общим) верхним уровнем $(N K)$ и переходами вниз только во второй яме на уровень с номером $L$ (рис. 1,c). Данная ситуация, на первый взгляд, практически полностью идентична случаю двухбарьерной структуры, однако на самом деле довольно сильно отличается. По крайне мере и в этом случае ширину верхнего уровня можно сделать меньше ширины нижнего.

Нестационарное уравнение Шредингера для рассматриваемых систем имеет вид

$$
\begin{aligned}
i \hbar \frac{\partial \psi}{\partial t}= & -\frac{\hbar^{2}}{2 m^{*}} \frac{\partial^{2} \psi}{\partial x^{2}}+H(x) \psi+H(x, t) \psi, \\
H(x)= & U_{1}[\theta(x)-\theta(x-a)] \\
& +U_{2} \theta(x-a)+\alpha \delta(x)+\alpha \gamma \delta(x-a), \\
H(x, t)= & -q E\left(e^{i \omega t}+e^{-i \omega t}\right) \\
& \times[x[\theta(x)-\theta(x-a)]+a \theta(x-a)]
\end{aligned}
$$

для двухбарьерных структур и

$$
\begin{aligned}
H(x)= & U_{1}[\theta(x)-\theta(x-a)]+U_{2}[\theta(x-a) \\
& -\theta(x-a-l)]+U_{3} \theta(x-a-l)+\alpha \delta(x) \\
& +\alpha \rho \delta(x-a)+\alpha \gamma \delta(x-a-l), \\
H(x, t)=- & q E\left(e^{i \omega t}+e^{-i \omega t}\right)\{x[\theta(x)-\theta(x-a-l)] \\
& +(a+l) \theta(x-a-l)\}
\end{aligned}
$$

для трехбарьерных. Здесь $q, m^{*}$ - заряд и масса электрона, $\alpha=\varphi_{b} b-$ мощность первого барьера, $\varphi_{b}, b-$ его высота и ширина, $\theta(x)$ - единичная функция, $\gamma, \rho-$ численные коэффициенты, равные отношению мощности соответствующего барьера к мощности барьера на входе структуры, $a, l$ - расстояния между барьерами, $U, U_{1}, U_{2}$ - величины скачков дна зоны проводимости на барьерах.
Для структур на рис. $1, a, b, d$ решения задачи при частоте электрического поля, строго соответствующей резонансной частоте переходов между центрами уровней, были получены в работах $[3,16]$, и имеют вид

$$
\begin{gathered}
\psi(x, t)=\psi_{K}(x) e^{-i \omega_{0} t}+\psi_{L}(x) e^{-i\left(\omega_{0}-\omega\right) t}, \\
\psi_{K}(x)=\left\{\begin{array}{l}
\exp \left(i k_{0} x\right)+D \exp \left(-i k_{0} x\right), x<0 \\
A \sin \left(k_{1} x\right)+B \cos \left(k_{1} x\right), 0<x<a \\
C \exp \left[i k_{3}(x-a)\right], x>a
\end{array}\right. \\
\psi_{L}(x)=F\left\{\begin{array}{l}
D_{-} \exp (\kappa x), x<0 \\
A_{-} \sin \left(k_{1-} x\right)+B_{-} \cos \left(k_{1-} x\right), 0<x<a \\
C_{-} \exp \left[i k_{3-}(x-a)\right], x>a
\end{array}\right.
\end{gathered}
$$

для двухбарьерной и

$$
\begin{aligned}
& \psi_{K}(x)= \\
& =\left\{\begin{array}{l}
\exp \left(i k_{0} x\right)+D \exp \left(-i k_{0} x\right), \quad x<0 \\
A \sin \left(k_{1} x\right)+B \cos \left(k_{1-}\right), 0<x<a \\
Z \sin \left[k_{2}(x-a)\right]+W \cos \left[k_{2}(x-a)\right], \quad a<x<a+l \\
C \exp \left[i k_{3-}(x-a-l)\right], x>a+l
\end{array}\right. \\
& \psi_{L}(x)=F \\
& \times\left\{\begin{array}{l}
D_{-} \exp (\kappa x), x<0 \\
A_{-} \sin \left(k_{1-} x\right)+B{ }_{-} \cos \left(k_{1} x\right), 0<x<a \\
Z_{-} \sin \left[k_{2-}(x-a)\right]+W_{-} \cos \left[k_{2}(x-a)\right], a<x<a+l \\
C_{-} \exp \left[i k_{3-}(x-a-l)\right], x>a+l
\end{array}\right.
\end{aligned}
$$

для трехбарьерной структуры. Данные волновые функции приведены для структур, в которых электроны по нижнему уровню уходят только направо (рис. $1, a-c$ ); для структур с возможностью ухода налево (рис. $1, d, e)$ в области $x<0$ константа затухания $\kappa$ для волновой функции нижнего уровня заменяется на $i k_{0-}$.

При достаточно мощных барьерах (а только при таких барьерах вообще и имеет смысл говорить о системе с резонансными уровнями) в отсутствие переменного электрического поля $(F=0)$ коэффициенты волновой функции (4) для двухбарьерной структуры равны

$$
\begin{gathered}
B_{0}=\beta=\frac{\beta_{0}}{1+i \delta \varepsilon / \Gamma_{K}}, \beta_{0}=\frac{2 \gamma^{2} k_{0}}{\gamma^{2} k_{0}+k_{3}}=1 \pm \sqrt{R_{0}}, \\
A_{0}=\frac{y}{k_{1}} \beta, C_{0}=\frac{\beta}{\gamma}(-1)^{K+1}, D_{0}=B_{0}-1,
\end{gathered}
$$

а коэффициенты волновой функции (5) для трехбарьерной структуры (рис. $1, b)$ равны

$$
\begin{gathered}
B_{0}=\beta=\frac{\beta_{0}}{1+i \delta \varepsilon / \Gamma_{K}}, \beta_{0}=2, A_{0}=2 y / k_{1}, \\
W_{0}=\frac{\beta(-1)^{K}}{\rho}, Z_{0}=\frac{\beta \operatorname{ctg}\left(k_{1} l\right)}{\rho}, C_{0}=-\frac{\beta k_{1}}{\gamma \rho y \sin k_{2} l} .
\end{gathered}
$$

Здесь и далее $K, L=1,2 \ldots$ 
Для трехбарьерной структуры на рис. 1, $c$ условия резонансного прохождения электронов по верхнему уровню есть

$$
\begin{gathered}
\cos k_{1} a \approx(-1)^{N}, \quad \cos k_{2} l \approx(-1)^{K}, \\
\sin k_{1-} a \approx-\frac{k_{1}}{y} \xi, \quad \sin k_{2-} l \approx-\frac{k_{2}}{y} \Omega, \\
\xi=\frac{1+\rho+\gamma+\gamma \Omega-(-1)^{K} \gamma \Omega \rho}{\gamma+\rho+(-1)^{K+1} \gamma \Omega \rho}(-1)^{L},
\end{gathered}
$$

а коэффициенты волновой функции в отсутствие электрического поля имеют вид

$$
B_{0}=\beta=\frac{\beta_{0}}{1+i \delta \varepsilon / \Gamma_{K}},
$$

$$
\beta_{0} \frac{2 k_{0}\left[\gamma+\rho+(-1)^{K} \gamma \Omega \rho\right]^{2}}{k_{2}+k_{0}\left[\gamma+\rho-(-1)^{K} \gamma \Omega \rho\right]^{2}}=1 \pm \sqrt{R_{0}}, \quad A=\beta \frac{y}{k_{1}},
$$$$
Z_{0}=\frac{\beta \gamma y}{k_{2}\left[\gamma+\rho-(-1)^{K} \gamma \Omega \rho\right]}, W_{0}=\frac{\beta(1+\gamma \Omega)}{\left[\gamma+\rho-(-1)^{K} \gamma \Omega \rho\right]},
$$

$$
C_{0}=\frac{\beta}{\left[\gamma+\rho-(-1)^{K} \gamma \Omega \rho\right]}, \quad D_{0}=\beta-1 .
$$

Здесь и далее $R_{0}$ - коэффициент отражения от центра верхнего уровня в отсутствие СВЧ поля, $k_{i}-$ соответствующие волновые векторы на каждом уровне и в соответствующей области пространства, $y=2 m^{*} \alpha \hbar^{2}-$ величина, которая имеет размерность волнового вектора. При достаточно мощных барьерах и соответственно узких резонансных уровнях $y \gg k_{i}$ и ряд теории возмущений строится по малому параметру $k_{i} / y$.

Анализ полученных выражений показывает, что трехбарьерная структура (рис. 1,c) становится абсолютно прозрачной при выполнении условий

$$
\Omega=-\left( \pm \frac{\sqrt{k_{3}}}{\sqrt{k_{0}}}+\gamma+\rho\right) / \gamma \rho, \xi=\left( \pm \frac{\sqrt{k_{0}}}{\sqrt{k_{2}}} \gamma+\rho\right) / \rho .
$$

Интересно отметить, что данные условия, описывающие два расщепленных уровня, оказываются условиями точного резонанса в полностью непрозрачной трехбарьерной структуре (когда дно зоны проводимости справа выше резонансного уровня $N K$ ).

Надо отметить важную особенность: в двухбарьерной структуре, при выполнении условия

$$
\gamma^{2} k_{0} \gg k_{3}, \beta_{0} \approx 2
$$

как и в трехбарьерной структуре, можно добиться полного отражения, однако ширина нижнего уровня при этом всегда будет меньше, чем верхнего. В трехбарьерной структуре ширину верхнего уровня можно менять независимо от нижнего и легко сделать его даже намного уже.
Условия резонанса для структур, в которых электроны уходят по нижнему уровню только направо (рис. $1, a, b)$, задаются условиями

$$
\begin{gathered}
\cos k_{1-} a \approx(-1)^{L}, \cos k_{2-} l \approx(-1)^{N}, \\
\sin k_{1-} a \approx-\frac{k_{1-}}{y}\left(\eta+\frac{\Delta \eta}{y}\right), \\
\sin k_{2-} l \approx-\frac{k_{2-}}{y}\left(\xi+\frac{\Delta \xi}{y}\right), \\
\eta=\frac{1+\rho+\gamma+\gamma \xi+(-1)^{L+1} \gamma \xi \rho}{\gamma+\rho+(-1)^{L+1} \gamma \xi \rho}(-1)^{L}, \\
\Delta \eta=(-1)^{L+1} \kappa-\frac{\gamma^{2} \Delta \xi}{\left[\gamma+\rho+(-1)^{L+1} \gamma \xi \rho\right]^{2}}
\end{gathered}
$$

для трехбарьерной структуры и

$$
\eta=\frac{1+\gamma}{\gamma}(-1)^{L}, \Delta \gamma=(-1)^{L+1} \kappa
$$

для двухбарьерной. Для трехбарьерной конкретные размеры структуры задают дополнительное соотношение между величинами $\eta$ и $\xi$, которое совместно с уравнением (11) определяет положение расщепленных уровней и расстояние между ними.

Для случаев, представленных на рис. $1, d, e$, условия резонанса имеют вид $\Delta \eta=\Delta \xi=0$.

Коэффициенты волновой функции на нижнем уровне, рассчитанные в первом порядке теории возмущений (см. подробнее [5]), для структур без отражения по нижнему уровню имеют вид

$$
\begin{gathered}
D_{-}=\beta_{-}=\frac{\beta_{0-}}{1+i \delta \varepsilon / \Gamma_{L}}, \\
A_{-}=\frac{y}{k_{1-}} \beta_{-}, B_{-}=\beta_{-}=\gamma C_{-}(-1)^{L+1} . \\
\beta_{0-}=\frac{q E}{i m^{*} \omega^{2}} \frac{2 \gamma^{2} y^{2}}{k_{3-}} \beta
\end{gathered}
$$

для двухбарьерной структуры,

$$
\begin{gathered}
D_{-}=\beta_{-}=\frac{\beta_{0-}}{1+i \delta \varepsilon / \Gamma_{L}}=B_{-}=A_{-} \frac{k_{-}}{y}, \\
C_{-}=\frac{\beta_{-}}{\left[\gamma+\rho+(-1)^{N+1} \gamma \xi \rho\right]}=Z_{-} \frac{k_{1-}}{\gamma y}=\frac{W_{-}}{1-\gamma \xi}, \\
\beta_{0-}=\frac{2 q E y^{2}\left[\gamma+\rho+(-1)^{N+1} \gamma \xi \rho\right]^{2}}{i m^{*} \omega^{2} k_{3}} \beta
\end{gathered}
$$

для трехбарьерной структуры на рис. $1, b$ и

$$
\begin{gathered}
C_{-}=\beta_{-}=\left(\frac{q E}{m^{*} \omega^{2}}\right) \frac{i \beta_{0} \gamma^{2} y^{2}}{k_{3-}\left[\gamma+\rho-(-1)^{K} \gamma \Omega \rho\right]}, \\
Z_{-}=-\beta \frac{\gamma y}{k_{2-}}, W_{-}=-\beta \gamma, \\
A_{-}=-\beta \frac{\rho \sin k_{1-} a}{\gamma}, D_{-}=-\beta \frac{\gamma k_{1-}}{\rho y \sin k_{1-} a}, \\
B_{-}=\frac{i \beta\left\{i k_{1-} \gamma^{3}+k_{3-} \rho \sin k_{1-} a\left[\gamma+\rho-(-1)^{K} \gamma \Omega \rho\right]\right\}}{y \gamma^{2} \rho \sin k_{1-} a}
\end{gathered}
$$

для трехбарьерной структуры на рис. 1,c. 
Для структур с открытым в обе стороны нижним уровнем (см. рис. $1, d, e)$

$$
\begin{gathered}
D_{-}=\beta_{-}=\frac{\beta_{0-}}{1+i \delta \varepsilon / \Gamma_{L}}, A_{-}=\frac{y}{k_{1-}} \beta_{-}, \\
B_{-}=\beta_{-}=\gamma C_{-}(-1)^{L+1}, \beta_{0-}=\frac{q E}{i m^{*} \omega^{2}} \frac{2 \gamma^{2} y^{2}}{\gamma^{2} k_{0-}+k_{3-}} \beta, \\
z_{0}=\left(\frac{q E}{m^{*} \omega^{2}}\right)^{2} \frac{4 \gamma^{4} y^{4}}{\left(\gamma^{2} k_{0}+k_{3}\right)\left(\gamma^{2} k_{0-}+k_{3-}\right)}
\end{gathered}
$$

для двухбарьерной [3] и

$$
\begin{gathered}
D_{-}=\beta_{-}=\frac{\beta_{0-}}{1+i \delta \varepsilon / \Gamma_{L}}=B_{-}=A_{-} \frac{k_{1-}}{y}, \\
\beta_{0-}=\frac{2 q E y^{2}\left[\gamma+\rho+(-1)^{N+1} \gamma \xi \rho\right]^{2}}{i m^{*} \omega^{2}\left\{k_{3-}+k_{0-}\left[\gamma+\rho+(-1)^{N+1} \gamma \xi \rho\right]^{2}\right\}} \beta, \\
C_{-}=\frac{\beta_{-}}{\left[\gamma+\rho+(-1)^{L+1} \gamma \xi \rho\right]}=Z_{-} \frac{k_{1-}}{\gamma y}=\frac{W_{-}}{1-\gamma \xi}, \\
z_{0}=\left(\frac{2 q E}{m^{*} \omega^{2}}\right)^{2} \frac{\left[\gamma+\rho+(-1)^{N+1} \gamma \xi \rho\right]^{2} y^{4}}{k_{0}\left\{k_{3-}+k_{0-}\left[\gamma+\rho+(-1)^{N+1} \gamma \rho\right]^{2}\right\}}
\end{gathered}
$$

для трехбарьерной структуры.

Данные задачи допускают получение решений в сильном поле на основе суммирования ряда теории возмущений (см., например, $[3,5])$. В результате имеем

$$
\begin{gathered}
B=\frac{B_{0}}{1+z}, A=\frac{A_{0}}{1+z}, C=\frac{C_{0}}{1+z}, Z=\frac{Z_{0}}{1+z}, \\
W=\frac{W_{0}}{1+z}, D=B-1, F=\frac{1}{1+z}, \\
z=z_{0} \frac{1}{1+i \delta \varepsilon / \Gamma_{L}} \frac{1}{1+i \delta \varepsilon / \Gamma_{K}},
\end{gathered}
$$

где для структур с уходом по нижнему уровню только направо (рис. $1, a-c$ )

$$
\begin{gathered}
z_{0}=\left(\frac{q E}{m^{*} \omega^{2}}\right)^{2} \frac{4 \gamma^{4} y^{4}}{k_{3-}\left(\gamma^{2} k_{0}+k_{3}\right)}, \\
z_{0}=\left(\frac{2 q E}{m^{*} \omega^{2}}\right)^{2} \frac{\left[\gamma+\rho+(-1)^{L+1} \gamma \xi \rho\right]^{2} y^{4}}{k_{0} k_{3-}}, \\
z_{0}=\left(\frac{q E}{m^{*} \omega^{2}}\right)^{2} \frac{2 y^{4} \gamma^{4}}{k_{2-}\left\{k_{2}+k_{0}\left[\gamma+\rho-(-1)^{K} \gamma \Omega \rho\right]^{2}\right\}},
\end{gathered}
$$

а для структур с отражением по нижнему уровню (рис. $1, d, e$ )

$$
\begin{gathered}
z_{0}=\left(\frac{q E}{m^{*} \omega^{2}}\right)^{2} \frac{4 \gamma^{4} y^{4}}{\left(\gamma^{2} k_{0}+k_{3}\right)\left(\gamma^{2} k_{0-}+k_{3-}\right)}, \\
z_{0}=\left(\frac{2 q E}{m^{*} \omega^{2}}\right)^{2} \frac{\left[\gamma+\rho+(-1)^{N+1} \gamma \xi \rho\right]^{2} y^{4}}{k_{0}\left\{k_{3-}+k_{0-}\left[\gamma+\rho+(-1)^{N+1} \gamma \xi \rho\right]^{2}\right\}} .
\end{gathered}
$$

Надо отметить, что данные решения применимы как в области сходимости соответствующих рядов, так и далеко за ее пределами [24].
Не представляет труда обобщить эти результаты на частоты, близкие к резонансной, при сдвиге на $\Delta \omega$ (в малом сигнале это было сделано в работе [20]). В этом случае решение задачи принимает вид

$$
\begin{gathered}
\beta_{-}=\frac{\beta_{0-}}{1+i(\delta \varepsilon-\Delta \omega \hbar) \delta \varepsilon / \Gamma_{L}}, \\
z=z_{0} \frac{1}{1+i \delta \varepsilon / \Gamma_{K}} \frac{1}{1+i(\delta \varepsilon-\Delta \omega \hbar) \delta \varepsilon / \Gamma_{L}} .
\end{gathered}
$$

Выражение (22) справедливо как для двухбарьерной, так и для трехбарьерной структуры, если заменить $\Gamma_{L}$ на $\Gamma_{L N}$. Здесь и далее в том случае, когда нет принципиальной разницы в обозначениях для двухбарьерной и трехбарьерной структур, вместо индекса $L N$ будем ставить просто индекс $L$, если отличия есть, индексы, естественно, будут выписываться полностью.

Выражения для ширин уровней в несимметричных двухбарьерных структурах приведены в (5). Аналогичным способом можно получить выражения для полуширины уровня для структуры, приведенной нв рис. $1, e$, в которой электроны могут уходить с общего уровня в обе стороны,

$$
\Gamma_{L N}=\Gamma_{\text {sim }}^{L} \frac{k_{2}+k_{0}\left[\gamma+\rho+(-1)^{L+1} \gamma \eta \rho\right]^{2}}{k\left\{\gamma^{2} L^{2} l^{3} / N^{2} a^{3}+\left[\gamma+\rho+(-1)^{L+1} \gamma \eta \rho\right]^{2}\right\}},
$$

и для структуры на рис. $1, b$, в которой электроны могут уходить с общего уровня только в одну сторону,

$$
\Gamma_{L N}=\Gamma_{\text {sim }}^{L} \frac{k_{2}}{k\left\{\gamma^{2} L^{2} l^{3} / N^{2} a^{3}+\left[\gamma+\rho+(-1)^{L+1} \gamma \eta \rho\right]^{2}\right\}} .
$$

Здесь $\Gamma_{\text {sim }}^{L}-$ полуширина уровня в симметричной структуре шириной $a \mathrm{c} \delta$-образными барьерами с мощностью $\alpha$ и высотой уровня $\Sigma_{L}$ от дна зоны проводимости (5):

$$
\Gamma_{\text {sim }}^{L}=\frac{4 k^{2} \Sigma_{L}}{\pi L y^{2}}, \quad \Sigma_{L}=\frac{\pi^{2} \hbar^{2} L^{2}}{2 m^{*} a^{2}}, \quad \Sigma_{N}=\frac{\pi^{2} \hbar^{2}}{2 m^{*} l^{2}} .
$$

\section{3. Расчет коэффициентов отражения и прохождения}

Падая на верхний уровень двухуровневой системы с приложенным высокочастотным полем, электроны частично проходят через нее (уходят направо как по верхнему, так и по нижнему уровню) и частично отражаются (уходят налево по верхнему и, если это возможно (см. рис. $1, d, e)$, по нижнему уровню).

В ситуации, когда электроны уходят налево (отражаются от структуры) только по верхнему уровню, довольно естественно определить коэффициент отражения классически как отношение отраженного потока $\left(J_{R}\right)$ к падающему $\left(J_{0}\right)$. При выбранной нормировке волновой функции это просто $|D|^{2}$. Аналогично можно определить коэффициент прохождения по верхнему уровню как 
отношение потока электронов, уходящих из структуры без изменения энергии направо $\left(J_{T}\right)$, к падающему $\left(J_{0}\right)$, и коэффициент прохождения по нижнему уровню как отношение потока электронов, уходящих из структуры направо и отдавших квант энергии $\hbar \omega\left(J_{T-}\right)$, к падающему, соответственно коэффициент отражения по нижнему уровню для электронов, отдавших квант энергии и уходящих налево, есть $\left(J_{R-}\right)$.

В общем случае коэффициент отражения от верхнего уровня структуры в сильном СВЧ поле для всех рассматриваемых структур имеет вид

$$
\begin{gathered}
R=1-\frac{\beta_{0} \Gamma_{K}^{2}\left\{2 z_{0} \Gamma_{L}^{2}+\left(2-\beta_{0}\right)\left[\Gamma_{L}^{2}+(\delta \varepsilon-\hbar \Delta \omega)^{2}\right]\right\}}{P(\delta \varepsilon, \Delta \omega)} \\
P(\delta \varepsilon, \Delta \omega)=\delta \varepsilon^{4}-2 \delta \varepsilon^{3} \hbar \Delta \omega-2 \delta \varepsilon \hbar \Delta \omega \Gamma_{K}\left(\Gamma_{K}-z_{0} \Gamma_{L}\right) \\
+\delta \varepsilon^{2}\left(\Gamma_{K}^{2}-2 z_{0} \Gamma_{K} \Gamma_{L}+\Gamma_{L}^{2}+\hbar^{2} \Delta \omega^{2}\right) \\
+\Gamma_{K}^{2}\left[\hbar^{2} \Delta \omega^{2}+\left(1+z_{0}\right)^{2} \Gamma_{L}^{2}\right] .
\end{gathered}
$$

Используя условие $\beta_{0}=1 \pm \sqrt{R_{0}}$, эту формулу легко переписать только через энергии, ширины уровней и коэффициент отражения. В этом случае знак перед радикалом будет определяться внутренними свойствами структуры: при $\beta_{0}>1$ он положителен, при $\beta_{0}<1$ отрицателен. Имеем:

$R=1-\frac{\Gamma_{K}^{2}\left\{2 z_{0} \Gamma_{L}^{2}\left(1 \pm \sqrt{R_{0}}\right)+\left(1-R_{0}\right)\left[\Gamma_{L}^{2}+(\delta \varepsilon-\hbar \Delta \omega)^{2}\right]\right\}}{P(\delta \varepsilon, \Delta \omega)}$.

Надо особо отметить, что решение несимметрично относительно ширин верхнего и нижнего уровней. Симметрия наступает только при полном отражении и строгом резонансе $\beta_{0}=2, \Delta \omega=0$ или тривиальном случае туннелирования на резонансной частоте строго через центр уровня.

В случае, когда взаимодействующие с полем электроны могут уходить только направо, отношение потоков по нижнему и верхнему уровням есть

$$
\frac{J_{T-}}{J_{T}}=\frac{k_{3-}\left|C_{-}\right|^{2}}{k_{3}\left|C_{0}\right|^{2}}=z_{0} \frac{\Xi}{1 \mp \sqrt{R_{0}}}\left|\frac{\Gamma_{L}}{\Gamma_{L}+i(\delta \varepsilon-\Delta \omega \hbar)}\right|^{2},
$$

где $\Xi=1$ для трехбарьерной структуры, рис. $1, c$, и $\Xi=2$ для двухбарьерной структуры, рис. 1, $a$. Отсюда сразу получаем

$$
T=\frac{(1-R)\left(1 \mp \sqrt{R_{0}}\right)}{1 \mp \sqrt{R_{0}}+\Xi z_{0} \Gamma_{L}^{2} /\left[\Gamma_{L}^{2}+(\delta \varepsilon-\Delta \omega \hbar)^{2}\right]}
$$

для коэффициента прохождения по верхнему уровню и

$$
T_{-}=\frac{\Xi z_{0}(1-R)}{\Xi z_{0}+\left(1 \mp \sqrt{R_{0}}\right)\left[1+(\delta \varepsilon-\Delta \omega \hbar)^{2} / \Gamma_{L}^{2}\right]}
$$

для коэффициента прохождения по нижнему уровню.

Естественно, для структуры на рис. $1, b$ автоматически получается, что все электроны проходят через структуру, только взаимодействуя с СВЧ полем.
Надо отметить очень интересный и принципиальный факт - коренное различие для данных задач между тонкими и толстыми (мощными) барьерами. Для электронов, падающих на структуру точно с энергией, соответствующей центру уровня,

$$
D=\frac{\beta_{0}}{1+z}-1, \beta_{0}=\frac{2 \gamma^{2} k_{0}}{\gamma^{2} k_{0}+k_{3}}=1 \pm \sqrt{R_{0}}
$$

При достаточно мощном барьере на выходе, в том плане что $\gamma^{2} k_{0}>k_{3}$, величина параметра $\beta_{0}$ оказывается $>1$. В этом случае включение резонансного СВЧ поля ведет к росту коэффициента прохождения. При $\gamma^{2} k_{0}<k_{3}$ ситуация меняется (в этом случае $\sqrt{R_{0}}$ во всех формулах надо брать с обратным знаком). Видно, что у двух структур при одинаковом коэффициенте отражения могут быть абсолютно разные СВЧ свойства: структура, в которой $\gamma^{2} k_{0}>k_{3}$, может стать абсолютно прозрачной при

$$
z=\frac{\gamma^{2} k_{0}-k_{3}}{\gamma^{2} k_{0}+k_{3}}
$$

а структура, имеющая тот же коэффициент отражения при волновом векторе на выходе из структуры $\tilde{k}_{3}$, равном

$$
\tilde{k}_{3}=\frac{\gamma^{4} k_{0}^{2}}{k_{3}},
$$

не станет прозрачной никогда. Надо отметить, однако, что в отсутствие СВЧ поля эти структуры, вообще говоря, не идентичны. Полуширина уровня в первом и втором случаях соответственно равны [5]

$$
\Gamma_{1}=\Gamma_{\operatorname{sim}} \frac{\gamma^{2} k_{0}+k_{3}}{2 \gamma^{2} k}, \Gamma_{2}=\Gamma_{\operatorname{sim}} \frac{\gamma^{2} k_{0}+\tilde{k}_{3}}{2 \gamma^{2} k},
$$

а их отношение равно

$$
\Gamma_{2} / \Gamma_{1}=\frac{\gamma^{2} k_{0}}{k_{3}} .
$$

С учетом неравенства $\gamma^{2} k_{0}>0$ видно, что в структурах, абсолютно прозрачных в СВЧ поле, ширина уровня всегда меньше, чем ширина соответствующего уровня в полупрозрачной структуре.

Качественно для двухбарьерных структур эффект вполне понятен: при „мощном“ барьере на входе структуры большинство падающих на структуру электронов от него просто отражается и в структуру не попадает, а следовательно, приложенное СВЧ поле увеличить коэффициент прохождения не может.

В то же время для трехбарьерной структуры на рис. 1, $c$ даже при $\gamma>1$ (мощном третьем барьере) существуют условия, когда коэффициент отражения не обращается в 0 в сильном резонансном СВЧ поле:

$$
\begin{aligned}
& -\frac{\gamma+\rho+\sqrt{k_{2} / k_{0}}}{\gamma \rho}<\Omega<-\frac{\gamma+\rho-\sqrt{k_{2} / k_{0}}}{\gamma \rho}, K=2 M, \\
& \frac{\gamma+\rho-\sqrt{k_{2} / k_{0}}}{\gamma \rho}<\Omega<\frac{\gamma+\rho+\sqrt{k_{2} / k_{0}}}{\gamma \rho}, K=2 M-1 .
\end{aligned}
$$


По-видимому, здесь принципиальную роль начинает играть средний барьер и местоположение системы расщепленных уровней. При выполнении условий (35) структура с мощным внешним барьером в СВЧ поле будет только уменьшать прозрачность.

В случае, когда электроны могут уходить по нижнему уровню как направо, так и налево, ситуация несколько усложняется, хотя выражение для коэффициента отражения от верхнего уровня (26) не меняется.

В отношении прохождения через структуру по верхнему уровню для подобных двухуровневых систем существует три заметно различающихся случая:

1) практически все электроны отражаются от верхнего уровня (коэффициент отражения от структуры велик, $\left.\beta_{0} \approx 2\right)$;

2) значительная часть электронов проходит через структуру по верхнему уровню (коэффициент отражения от структуры мал, $\beta_{0} \approx 1$, при этом $\beta_{0}>1$ );

$3)$ большая часть электронов отражается от первого барьера структуры (можно считать, внутрь структуры почти не попадает, $\left.\beta_{0}<1\right)$.

Третий случай малоинтересен. Рассмотрим первый случай, он наиболее интересен для практики. При полном отражении $\beta_{0} \approx 2$ для коэффициента прохождения получаем выражение (электроны уходят направо только по нижнему уровню):

$$
\begin{aligned}
T=4 z_{0} \Gamma_{K}^{2} \Gamma_{L}^{2} /\left[\delta \varepsilon^{4}\right. & -2 \delta \varepsilon^{3} \hbar \Delta \omega-2 \delta \varepsilon \hbar \Delta \omega \Gamma_{K}\left(\Gamma_{L}-z_{0} \Gamma_{L}\right) \\
+ & \delta \varepsilon^{2}\left(\Gamma_{K}^{2}-2 z_{0} \Gamma_{K} \Gamma_{L}+\Gamma_{L}^{2}+\hbar^{2} \Delta \omega^{2}\right) \\
+ & \left.\Gamma_{K}^{2}\left(\hbar^{2} \Delta \omega^{2}+\left(1+z_{0}\right)^{2} \Gamma_{K}^{2}\right)\right] .
\end{aligned}
$$

Известно, что при строго резонансной частоте с ростом амплитуды СВЧ поля такая структура становится абсолютно прозрачной в центре резонансного уровня $[2,5]$. При строго резонансной частоте и широком (по сравнению с нижним) верхнем уровне с увеличением амплитуды поля происходит расширение резонансного уровня, а коэффициент прохождения по центру уровня вначале доходит до единицы, а затем падает [16]. При этом максимальный коэффициент прохождения остается в центре уровня.

Гораздо интересней ситуация, когда ширины верхнего и нижнего уровня становятся сравнимы (рис. 2). В этом случае с ростом амплитуды поля уровень расширяется, затем меняет форму - становится плоским, а при дальнейшем увеличении амплитуды на коэффициенте прохождения появляется два максимума в точках

$$
\delta \varepsilon= \pm \frac{\sqrt{2 \Gamma_{K} \Gamma_{L} z_{0}-\Gamma_{K}^{2}-\Gamma^{2}}}{\sqrt{2}} .
$$

При этом

$$
|z|^{2}=\frac{4 z_{0}^{2} \Gamma_{K}^{2} \Gamma_{L}^{2}}{2 \Gamma_{K}^{2} \Gamma_{L}^{2}\left(1+2 z_{0}^{2}\right)-\Gamma_{K}^{4}-\Gamma_{L}^{4}},
$$

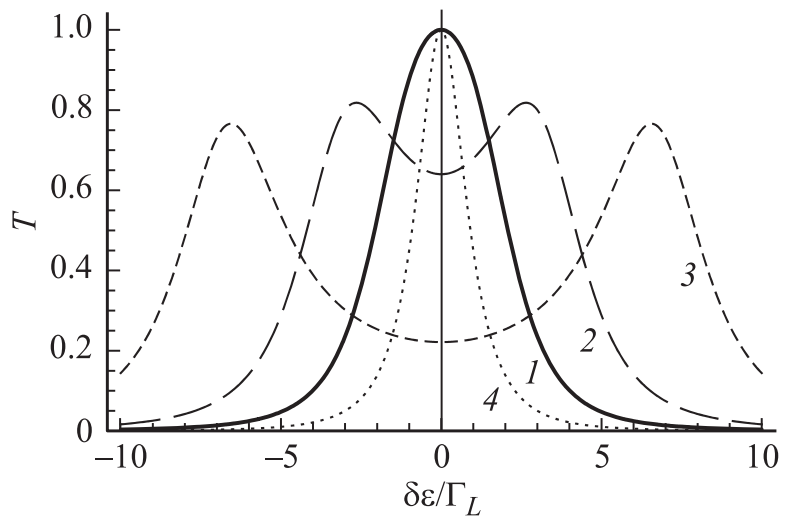

Рис. 2. Зависимости коэффициента прохождениия электронов от расстояния до центра резонансных уровней: $z_{0}=1(1)$, 4 (2), 16 (3). 4 - нормированная малосигнальная вероятность переходов. $\Gamma_{K}=3 \Gamma_{L}$.

а коэффициент прохождения в максимумах

$$
T=\frac{16 z_{0} \Gamma_{K}^{2} \Gamma_{L}^{2}}{\left[2 \Gamma_{K} \Gamma_{L}\left(1+2 z_{0}\right)-\Gamma_{K}^{2}-\Gamma_{L}^{2}\right] /\left(\Gamma_{L}+\Gamma_{K}\right)^{2}} .
$$

Еще интересней ситуация при равной ширине уровней (напомним, что такая ситуация возможна только для трехбарьерных структур) - там два максимума появляются практически сразу, причем в каждом, см. (39), коэффициент прохождения остается равным 1. Можно легко показать, что единичный коэффициент прохождения при строго резонансной частоте наблюдается только при равной ширине уровней $\Gamma_{L}=\Gamma_{L}$ и отклонении энергии от резонансной на величину

$$
\delta \varepsilon= \pm \Gamma_{K} \sqrt{z_{0}-1}
$$

при этом $|z|=1$.

С ростом амплитуды высокочастотного поля расстояние между максимумами увеличивается и в конце концов становится больше ширины уровня - один уровень расщепляется на два [16]. При этом ширины новых уровней равны

$$
\Gamma=\Gamma_{K}\left(\sqrt{z_{0}+2 \sqrt{z_{0}}-1}-\sqrt{z_{0}-2 \sqrt{z_{0}}-1}\right) .
$$

Естественно, это выражение имеет смысл, только когда подкоренное выражение во втором слагаемом $>0$, т.е. при $z_{0} \geq(1+\sqrt{2})^{2}=3+2 \sqrt{2}$. Надо отметить, что в сильных полях $\left(z_{0} \gg 1\right) \Gamma \approx 2 \Gamma_{K}$.

Наблюдается очень интересный эффект: из-за квантовой интерференции в сильном поле появляются два абсолютно прозрачных канала рассеяния, при энергиях электронов, существенно (более, чем на ширину линии) отличных от резонансной, - т.е. в сильных полях уровень расщепляется на два уровня вдвое большей ширины. 
Не менее интересно поведение коэффициента прохождения при частотах, отличных от резонансной. Анализируя (26), можно показать, что коэффициент прохождения становится равным при условии

$$
\hbar \Delta \omega=\frac{\begin{array}{c}
\delta \varepsilon^{3}+\delta \varepsilon \Gamma_{K}\left[\left(\beta_{0}-1\right)^{2} \Gamma_{K}-z_{0} \Gamma_{L}\right] \pm \\
\pm i \Gamma_{L}\left[\delta \varepsilon^{2}+\left(\beta_{0}-1\right)\left(\beta_{0}-z_{0}-1\right) \Gamma_{K}^{2}\right]
\end{array}}{\delta \varepsilon^{2}+\left(\beta_{0}-1\right)^{2} \Gamma_{K}^{2}} .
$$

Из требования равенства мнимой части нулю находим, что коэффициент прохождения равен 1 при отклонении энергии электронов от центра верхнего уровня

$$
\delta \varepsilon= \pm \Gamma_{K} \sqrt{\left(\beta_{0}-1\right)\left(z_{0}-\beta_{0}+1\right)}
$$

и сдвиге частоты от резонансной

$$
\hbar \Delta \omega= \pm \frac{\left|\left(\beta_{0}-1\right) \Gamma_{K}-\Gamma_{L}\right| \sqrt{z_{0}-\beta_{0}+1}}{\sqrt{\beta_{0}-1}} .
$$

Автоматически появляется требование на соотношение мощностей барьеров $\left(\beta_{0}>1\right)$. При этом, как и в случае одинаковой ширины уровней, $|z|=1$.
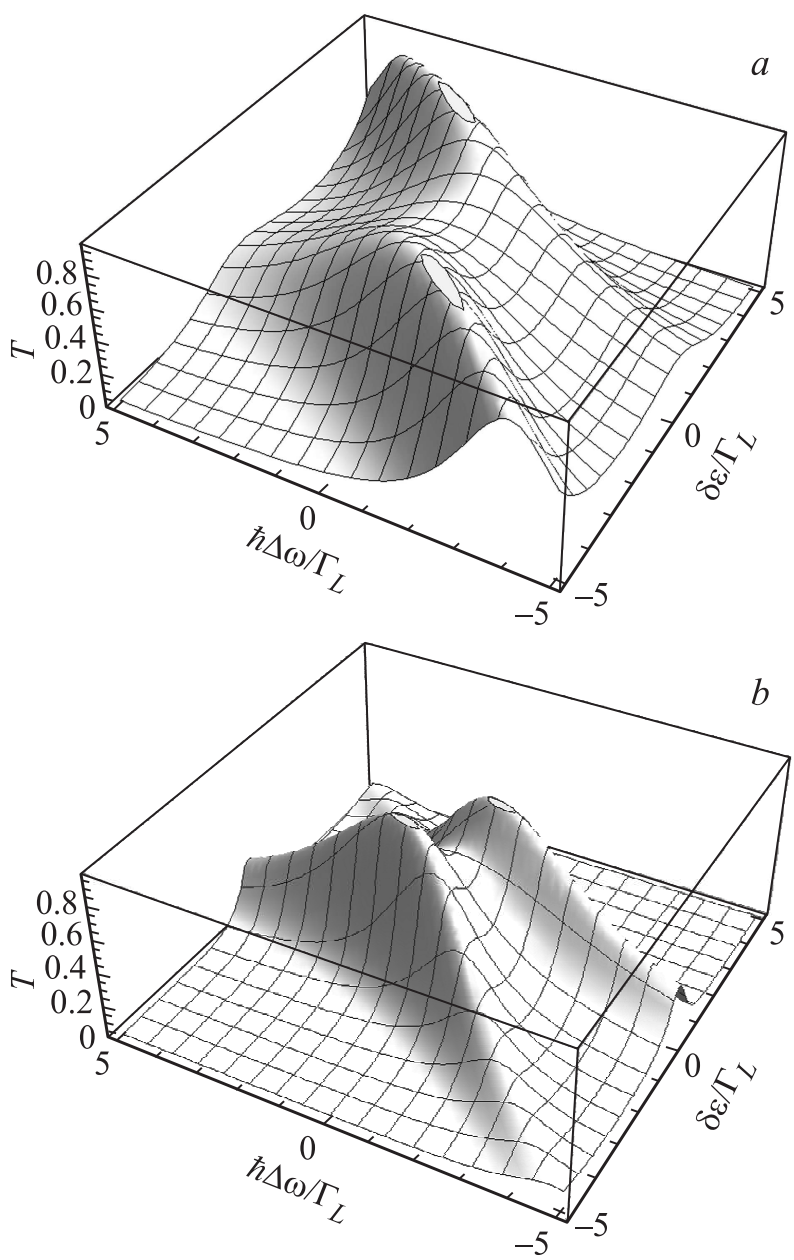

Рис. 3. Зависимости коэффициента прохождения от расстояния до центра уровней и сдвига частоты относительно резонансной. $z_{0}=3, R_{0}=1 ; \Gamma_{K}=2 \Gamma_{L}(a), \Gamma_{K}=0.5 \Gamma_{L}(b)$. Овалами выделены области, где коэффициент прохождения $>98 \%$.

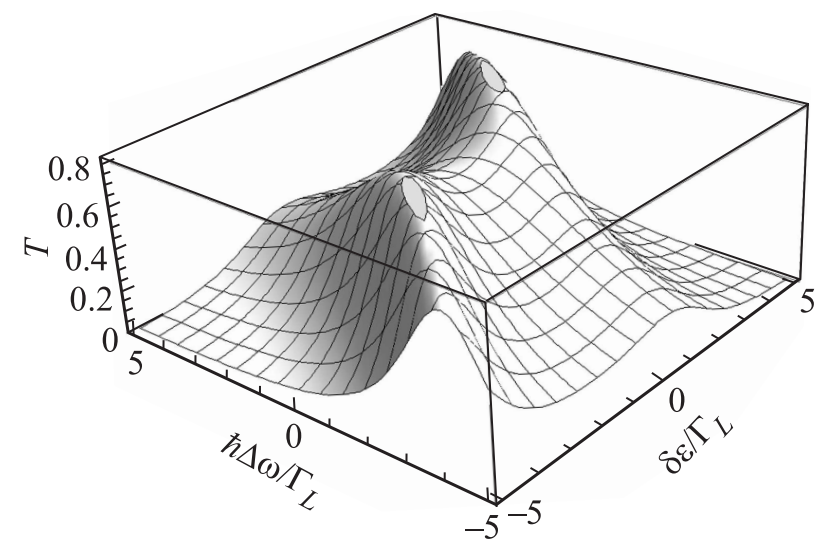

Рис. 4. Зависимость коэффициента прохождения по нижнему уровню двухбарьерной структуры от расстояния до центра уровней и сдвига частоты относительно резонансной. $z_{0}=3$, $R_{0}=0.5, \Gamma_{K}=2 \Gamma_{L}$. Овалами выделены области, где коэффициент прохождения $>85 \%$.

Таким образом, оказывается, что в двухуровневых системах с высокими тонкими барьерами при сравнимых ширинах резонансных уровней и баллистическом транспорте электронов для любой достаточно большой амплитуды СВЧ поля $\left(z_{0} \geq \sqrt{R_{0}}\right)$ с частотой, близкой к резонансной, существует близкая к резонансной энергия электронов (одна или две), при которой неупругий канал рассеяния становится абсолютно прозрачным.

Долгое время считалось, что ситуация, когда многобарьерная структура становится абсолютно прозрачной в СВЧ поле или хотя бы интенсивность переходов становится велика, - довольно экзотическая и трудно реализуемая. Данные оценки показывают, что ограничения на подобные эффекты гораздо более слабые, чем можно было бы ожидать, а поэтому создание квантовых каскадных лазеров с баллистическим транспортом электронов [11] - задача вполне выполнимая уже при существующем уровне технологии.

Как пример на рис. 3 приведены зависимости коэффициентов прохождения при полном статическом отражении $\left(R_{0}=1\right)$ при разных ширинах уровней. Овалами выделены области, в которых коэффициент отражения $<1 \%$, т.е. по сути дела области абсолютной прозрачности.

Видно, что области, в которых коэффициент прохождения велик (а значит, большое количество электронов отдало квант энергии), весьма широки. Это еще раз подтверждает вывод о том, что для наблюдения подобных эффектов, вполне возможно, не требуется сверхточного изготовления активных ячеек. Еще одним аргументом в пользу этой гипотезы служит рис. 4, на котором приведен коэффициент прохождения по нижнему уровню для полупрозрачной двухбарьерной структуры $\left(R_{0}=0.5\right)$.

Для трехбарьерной структуры на рис. 1,c коэффициент прохождения по нижнему уровню имеет практиче- 
ски тот же вид, что и на рис. 4, с той лишь разницей, что величина в максимуме чуть меньше $(0.75$, а не 0.85$)$.

Как отмечалось выше, одной из отличительных особенностей коэффициента прохождения через структуру в высокочастотном поле является асимметрия решения относительно ширин верхнего и нижнего уровней. Пример приведен на рис. $3, b$, где по отношению к рис. 3, $a$ ширины верхнего и нижнего уровней поменяны местами. Видна радикальная перестройка коэффициента прохождения (оси эллипсоидов прозрачности 98\% ориентированы не относительно диагонали, а почти параллельно оси $\left.\hbar \Delta \omega / \Gamma_{L}\right)$.

\section{4. Заключение}

Для несимметричных дувухбарьерных и трехбарьерных резонансно-туннельных структур с тонкими высокими барьерами, образующих открытые двухуровневые системы, получены аналитические выражения для коэффициентов прохождения и отражения электронов в сильном высокочастотном электрическом поле с частотой, близкой к резонансной. Исследованы зависимости ширины и формы резонансных уровней от амплитуды СВЧ поля. Показано, что для таких структур практически всегда существуют условия, при которых нерезонансные каналы рассеяния вблизи квантовых уровней могут становиться абсолютно прозрачными. Поэтому создание квантовых каскадных лазеров с баллистическим транспортом электронов, использующих подобные структуры в качестве активной ячейки, выглядит вполне выполнимой задачей уже при существующем уровне технологии

\section{Список литературы}

[1] M. Butiker, R. Landauer. Phys. Rev. Lett., 49(23), 1739 (1982).

[2] М.Ю. Сумецкий, М.Л. Фельштын. Письма ЖЭТФ, 53 (1), 24 (1991).

[3] Е.И. Голант, А.Б. Пашковский. Письма ЖЭТФ, 63 (7), 559 (1996).

[4] А. Гельвич, Е.И. Голант, И.В. Кузнецова, А.Б. Пашковский. Письма ЖТФ, 29(13), 64 (2003).

[5] Е.И. Голант, А.Б. Пашковский. ЖЭТФ, 112 (7), 237 (1997).

[6] В.Ф. Елесин. ЖЭТФ, 112 (8), 483 (1997).

[7] В.Ф. Елесин. ЖЭТФ, 122 (7), 131 (2002).

[8] T.C.L.G. Sollner, P.E. Goodhue, P.E. Tannenwald, C.D. Parker, D.D. Peck. Appl. Phys. Lett., 43 (6), 588 (1985).

[9] E.R. Brown, T.C.L.G. Sollner, C.D. Parker, W.D. Goodhue, C.L. Chen. Appl. Phys. Lett., 55 (17), 1777 (1989).

[10] А.Ф. Казаринов, Р.А. Сурис. ФТП, 6, 135 (1972).

[11] Е.И. Голант, А.Б. Пашковский, А.С. Тагер. Письма ЖТФ, 20 (21), 74 (1994)

[12] J. Faist, F. Capasso, D.L. Sivco. Science, 264, 553 (1994).

[13] S. Blaser, M. Rochat, M. Beck, J. Faist. Phys. Rev. B, 61 (12), 8369 (2000).

[14] C. Gmachl, F. Capasso, D.L. Sivco, A.Y. Cho. Rep. Prog. Phys., 64, 1533 (2001).
[15] M.A. Belkin, F. Capasso, F. Xie, A. Belyanin, M. Fischer, A. Wittmann, J. Faist. Appl. Phys. Lett., 92, 201101 (2008).

[16] А.Б. Пашковский. Письма ЖЭТФ, 89 (1), 32 (2009).

[17] А.Б. Пашковский. Письма ЖЭТФ, 93 (10), 620 (2011).

[18] Н.В. Ткач, Ю.А. Сети. Письма ЖЭТФ, 95 (5), 296 (2012).

[19] В.М. Галицкий, Б.М. Карнаков, В.И. Коган. Задачи по квантовой механике (М., Наука, 1981).

[20] И.В. Беляева, Е.И. Голант, А.Б. Пашковский. ФТП, 31 (2), 137 (1997).

[21] И.А. Обухов. Неравновесные эфбфекты в электронных приборах (М., Вебер, 2010).

[22] И.И. Абрамов. Нано- и микросистемная техника, 109 (8), 7 (2009).

[23] А.Б. Пашковский. Электронная техника. Сер. 1, СВЧ-техника, 492 (4), 17 (2007).

[24] Е.И. Голант, А.Б. Пашковский. Теоретическая и математическая физика, 120 (2), 332 (1999).

Редактор Л.В. Шаронова

\section{Peculiarities of ballistic electron thansport in double-levels systems at strong high frequency electric fields}

\section{A.B. Pashkovskii \\ Joint-Stock Company Research \& Production Corporation „ISTOK" named after A.I. Shokin, 141190 Fryazino, Moscow reg., Russia}

Abstract For asymmetric double-barrier and triple-barrier resonant-tunneling structures with thin and high barriers, the simple analytical expressions that describes resonant transitions, which happen in the large-signal high-frequency electric fields with close to resonance frequency has been obtained. The levels width dependence on amplitude of intensive high frequency electric field have been investigated. It is shown, that for this structures conditions when non-resonance scattering channel near quantum levels can be absolutely transparent exist almost invariably. 